\section{Two-Hybrid Selection Assay to Identify Proteins Interacting with Poly- merase II Transcription Factors and Regulators}

BioTechniques 30:296-302 (February 2001)

\section{ABSTRACT}

The RNA polymerase III-based two-hybrid system has been developed to detect interactions between proteins such as RNA polymerase II transcription factors and regulators that cannot be studied by the original RNA polymerase II two-hybrid system. This novel method appears to be most useful for a refined analysis of already known protein-protein interactions. However, the application of this system in library screenings has been impaired by the lack of a suitable assay for the selection of the activated pol III reporter gene in yeast. Here, we describe a novel selection assay for the pol III-based two-hybrid system that makes it readily usable for screening expression libraries to search for interacting partners. Our system utilizes a temperature-sensitive (ts) U6 snRNA, which is synthesized by RNA polymerase III from a mutated SNR6 gene in yeast. In this ts strain, interactions between hybrid proteins activate an artificial pol III reporter construct (UAS $G^{-S N R 6), ~}$ which controls expression of wild-type U6 snRNA. This wild-type U6 snRNA can suppress the ts phenotype and allow growth at the nonpermissive temperature of $37^{\circ} \mathrm{C}$, thus providing a positive selection system for interacting proteins.

\section{INTRODUCTION}

The yeast two-hybrid system is a powerful technique to identify novel protein-protein interactions; this technology is widely applied in the new field of proteomics (12-14). One limitation of the original system (4), which is based on the selective activation of reporter genes transcribed by RNA polymerase II (pol II), is that it is not suitable for studying transcription factors and regulators of RNA pol II; in- deed, most of these proteins activate the reporter genes on their own (i.e., in the absence of interacting partners) $(2,10)$. A solution to this problem could be provided by an alternative two-hybrid system developed by Marsolier et al. (10), which is based on activation of a modified SNR6 reporter gene $\left(\mathrm{UAS}_{\mathrm{G}^{-}}\right.$ SNR6) transcribed by RNA polymerase III (pol III). No pol II transcription factor has indeed been shown to be able to activate pol III genes (Reference 10 and unpublished data). The reporter construct of the pol III-based two-hybrid system, $\mathrm{UAS}_{\mathrm{G}^{-}}$SNR6, bears 5 Gal4pbinding sites $\left(\mathrm{UAS}_{\mathrm{G}}\right)$ in place of the original $\mathrm{B}$ block. This otherwise inactive $\mathrm{UAS}_{\mathrm{G}^{-}}$SNR6 reporter gene can be specifically activated by tethering the pol III-specific transcription factor $\tau 138$ p to DNA through a fusion with the Gal4p DNA-binding domain (DBD) [Gal4(1-147)- $138 \mathrm{p}]$ (9). Activation of $\mathrm{UAS}_{\mathrm{G}}-\mathrm{SNR6}$ can also be achieved by fusing $\tau 138$ p to a protein that interacts with a known partner fused to the Gal4pDBD (i.e., by a twohybrid interaction) (10).

In the original method provided by Marsolier et al. (10), a genetic assay for U6 snRNA synthesis is used to indirectly monitor expression of the $\mathrm{UAS}_{\mathrm{G}^{-}}$ SNR6 reporter gene. These authors have constructed an snr6 yeast strain harboring a wild-type SNR6 gene on a URA3-marked plasmid. Loss of this plasmid is tolerated by yeast cells when the $\mathrm{UAS}_{\mathrm{G}^{-}}$SNR6 reporter gene carried by a second plasmid is activated, thus providing the cells with the essential U6 snRNA. Due to the presence of the URA3 marker gene, whose product turns 5-fluorotic acid (5-FOA) into a toxic compound, yeast cells that have lost the wild-type SNR6 plasmid can be identified for their ability to grow on agar plates containing 5-FOA. However, this selection assay for the expression of the $\mathrm{UAS}_{\mathrm{G}^{-}}$SNR6 reporter gene turned out to be unsuitable for largescale screening experiments because it gave a high background of yeast colonies growing on 5-FOA plates independently of activation of the $\mathrm{UAS}_{\mathrm{G}^{-}}$ SNR6 reporter gene (unpublished observations and see below). We do not have an evident explanation for such a high background. We have, however, observed that yeast transformation with overexpressed cDNA libraries, as typically applied in a two-hybrid system, can generally cause reduced selectivity of the 5-FOA conditions and, consequently, to Ura3 ${ }^{+}$cells still capable of growing on 5-FOA (our unpublished observations). Thus, under these conditions, screening of expression libraries can lead to the isolation of a high number of false-positive clones. Moreover, 5-FOA selection is generally applicable only via replica plating, which makes large-scale screenings in any case extremely laborious. We have therefore developed a quick and reliable selection assay for the pol III-based two-hybrid system.

\section{MATERIALS AND METHODS}

\section{Yeast and Plasmids}

The interaction assays were performed in the yeast strain MPy11 (MATo, ura3-52, lys2-801, ade2-101, trp1- $\Delta 63$, his3- $\Delta 200$, leu2- $\Delta 1$, snr6$B \Delta 2$, gal4::kan ${ }^{r}$ ). This strain is a derivative of MCM616 (11) in which the endogenous SNR6 gene is silent and the original plasmid carrying wild-type SNR6 has been replaced by a URA3marked centromeric plasmid bearing the temperature-sensitive (ts) snr6 A59C mutant. The ts point mutation A59C (8) was introduced into the SNR6 gene by PCR. The DNA fragment containing the snr6 A59C locus from -139 to +313 was subcloned into the KpnI/HindIII-digested episomal vector YCplac33 (6).

To construct the $\mathrm{UAS}_{\mathrm{G}}$-SNR6 reporter pMP44, the HIS3-containing EcoRI/XhoI fragment of JJ215 was subcloned in pOT2, a plasmid carrying the CAT gene marker. The blunted BglI/ AccI $2-\mu \mathrm{m}$ fragment of YEplac195 was inserted into the EcoRV site of the HIS3-containing pOT2 vector. The resulting HIS3 $2-\mu \mathrm{m}$ shuttle vector was cut with BamHI and EcoRI, and a $\mathrm{UAS}_{\mathrm{G}}$-SNR6 BamHI/EcoRI fragment was cloned into it.

The plasmids expressing the $\tau 138$ hybrid proteins listed in Table 1 are based on the TRP1-marked pGen- $\tau 138$ vector (10), in which the $B$ amHI site $5^{\prime}$ of the $\tau 138$ ORF has been deleted (a gift from M. Hottiger, University of Zürich) and a hemagglutinin (HA)- 
epitope sequence has been inserted at the BamHI site immediately downstream of the $\tau 138$ ORF. Details of all the plasmids are available upon request.

\section{Interaction Assays}

The ts yeast strain MPy11 was transformed with the $\mathrm{UAS}_{\mathrm{G}}-\mathrm{SNR} 6$ reporter plasmid pMP44 and the plasmid expressing the $\tau 138$-hybrid protein (bait construct). This ts yeast strain was always kept at $25^{\circ} \mathrm{C}$ instead of $30^{\circ} \mathrm{C}$ to increase the temperature gap between selective and nonselective growth conditions. Four single transformants were picked, and about $10^{8}$ cells/transformant were plated and incubated at $37^{\circ} \mathrm{C}$ to test temperature sensitivity. The clone of choice (the one that gave the lowest number of colonies at $37^{\circ} \mathrm{C}$ ) was transformed with the LEU2-marked plasmids expressing Gal4p(1-100) or the Gal4pDBD-fusion protein of interest according to the standard LiOAc protocol (5) with the following modifications: yeast cells were always kept at $25^{\circ} \mathrm{C}$ instead of $30^{\circ} \mathrm{C}$ and heat-shocked in a $42^{\circ} \mathrm{C}$ water bath for only $10 \mathrm{~min}$. After quick centrifugation in a micro- centrifuge, the cell pellet was gently resuspended in $200 \mu \mathrm{L}$ sterile water. Each cell suspension was split and spread on two selective plates (-His/Trp/Leu) that were incubated at $25^{\circ} \mathrm{C}$ for $24 \mathrm{~h}$. One plate was then shifted to $37^{\circ} \mathrm{C}$, while the other remained at $25^{\circ} \mathrm{C}$ to estimate transformation efficiency. Two to three days after the shift at $37^{\circ} \mathrm{C}$, colonies of cells potentially expressing interacting proteins started to appear. These colonies were picked and sequentially streaked on plates containing $0.1 \% 5$ FOA and on -His/Trp/Leu drop-out plates. Colonies growing on 5-FOA plates were picked and further processed to isolate plasmids (7).

\section{RESULTS AND DISCUSSION}

Figure 1 shows the principle of our novel selection technique to identify interacting proteins in a pol III-based two-hybrid system. The SNR6 knockout strain MPy11 contains a ts U6 snRNA expressed from the mutant snr6 $A 59 C$ gene that is carried by a URA3marked plasmid (ts survival construct). This mutated U6 allows growth at $30^{\circ} \mathrm{C}$ or less, but not at $37^{\circ} \mathrm{C}$. A second plasmid carries the $\mathrm{UAS}_{\mathrm{G}^{-}}$SNR6 reporter gene (reporter). This reporter gene expresses wild-type U6 snRNA when RNA pol III transcription is activated by tethering $\tau 138 p$ to DNA, for example, through an interaction between protein $\mathrm{X}$ fused to $\tau 138$ and protein $\mathrm{Y}$ fused to the $\mathrm{UAS}_{\mathrm{G}}$-binding Gal4pDBD. Expression of the wild-type U6 snRNA suppresses the ts phenotype and allows cell growth at $37^{\circ} \mathrm{C}$. Such a selection for growth at the nonpermissive temperature of $37^{\circ} \mathrm{C}$ is very tight; it is also technically easy and convenient because it does not require a replica-plating step. Moreover, since the ts survival construct carries the $U R A 3$ gene marker, this system allows researchers to exploit growth selection on 5-FOA plates, not for the primary selection from millions of clones as in the original system, but for a second selection step to confirm interacting partners in individual cell clones. Those clones that can grow at $37^{\circ} \mathrm{C}$ because of true activation of the $\mathrm{UAS}_{\mathrm{G}}-\mathrm{SNR} 6$ reporter gene can be selected for the loss of this plasmid by testing their ability to grow on 5-FOA plates. This second step is particularly

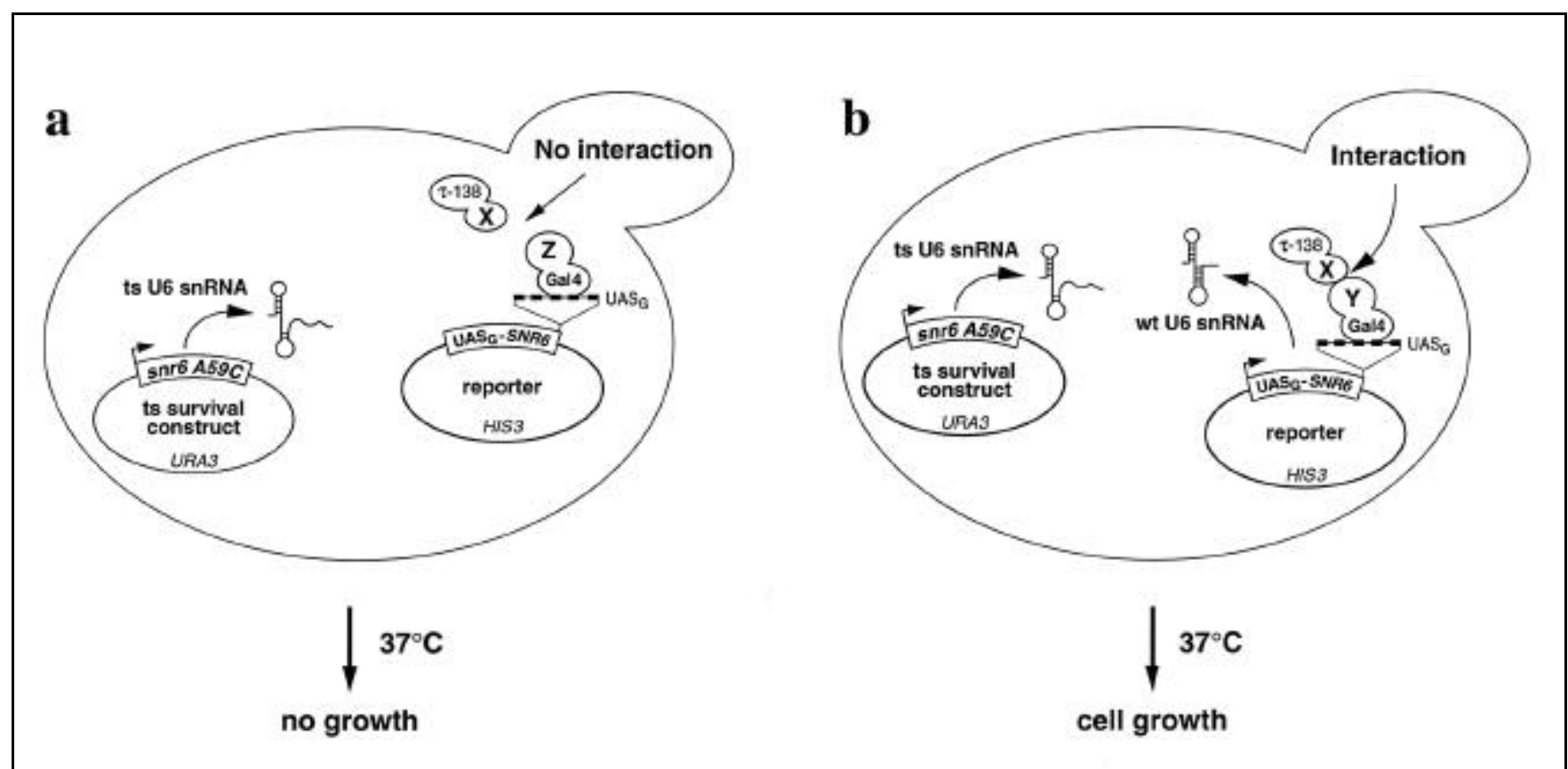

Figure 1. Outline of the RNA pol III two-hybrid selection assay. In the SNR6 knockout strain MPy11, the ts mutant snr6 A59Cgene is carried by a URA3marked plasmid (ts survival construct). A second plasmid carries the $\mathrm{UAS}_{\mathrm{G}}-S N R 6$ reporter gene (reporter). (A) The $\mathrm{UAS}_{\mathrm{G}}-S N R 6$ reporter gene is not activated if no interaction occurs between the fusion proteins $\tau 138-\mathrm{X}$ and Z-Gal4p; as a consequence, yeast cells cannot grow at $37^{\circ} \mathrm{C}$. (B) Activation of the $\mathrm{UAS}_{\mathrm{G}}-\mathrm{SNR} 6$ reporter gene caused by the interaction between the fusion proteins $\tau 138-\mathrm{X}$ and Y-Gal4p leads to expression of the wild-type copy of the U6 snRNA, which suppresses the ts phenotype (cell growth at $37^{\circ} \mathrm{C}$ ). For simplicity, RNA polymerase III and transcription factors other than $\tau 138$ have been omitted. 
useful for library screenings because it eliminates cells that contain mutations or hybrid proteins that may suppress the ts phenotype but are still dependent on the presence of the URA3-marked ts survival plasmid.

We first tested whether expression of
Gal4(1-147)- $\tau 138 p$ (10) would activate transcription of the $\mathrm{UAS}_{\mathrm{G}}-\mathrm{SNR} 6$ reporter gene to a level that is sufficient to sustain growth of the snr6 ts mutant strain at $37^{\circ} \mathrm{C}$. As shown in Figure 2 (plate b), expression of Gal4(1-147)$\tau 138 \mathrm{p}$ was able to suppress the snr6 ts

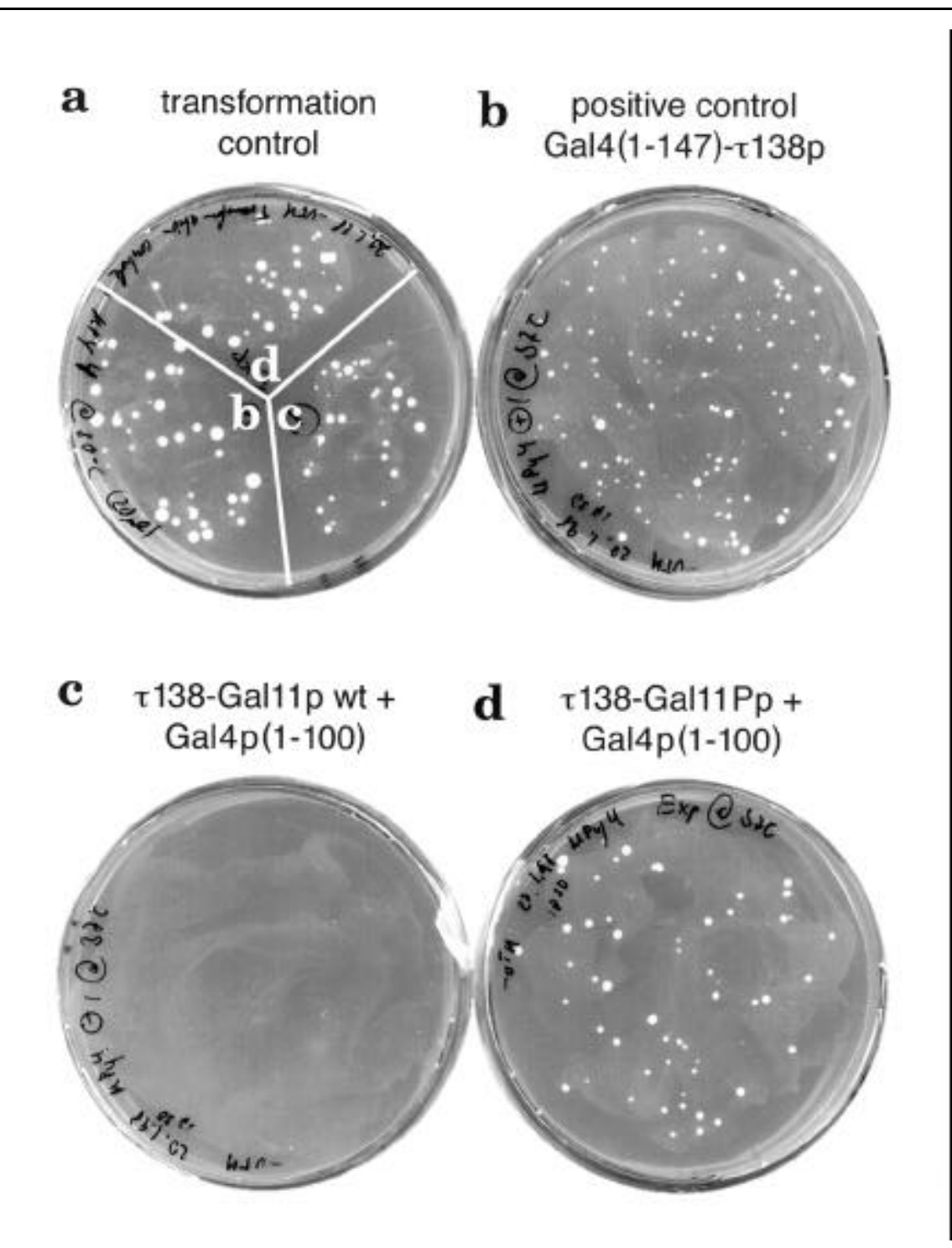

Figure 2. Suppression of the snr6 ts phenotype by interaction-dependent expression of wild-type U6 snRNA from the $\mathbf{U A S}_{\mathbf{G}^{-}}$SNR6 reporter gene. The $S N R 6$ knockout strain MPy11, which contains the $U R A 3$-marked ts survival construct and the $\mathrm{UAS}_{\mathrm{G}}-S N R 6$ reporter plasmid, was transformed with vectors expressing the Gal4(1-147)- $\tau 138 p$ fusion protein (b; positive control), $\tau 138-G a l 11 p$ wt plus $G a 14 p(1-100)$ (c), or $\tau 138-$ Gal11Pp plus Gal4p(1-100) (d). For each transformation, an aliquot of the cell suspension $(1 / 3$ of the volume) was spread in one of the three sectors of plate a, while the rest was spread on separate plates (plates b, c, and d). Plate a was incubated at $25^{\circ} \mathrm{C}$ for 4 days, while plates b, c, and d were incubated for $24 \mathrm{~h}$ at $25^{\circ} \mathrm{C}$ and then for 3 days at $37^{\circ} \mathrm{C}$. (Plate a) Transformation controls incubated at the permissive temperature of $25^{\circ} \mathrm{C}$. (Plate b) Expression of Gal4(1-147)- $\tau 138 p$ suppressed the ts phenotype and allowed cell growth at $37^{\circ} \mathrm{C}$. (Plate c) Co-expression of the noninteracting proteins $\tau 138$-Gal11p wt and Gal4p $(1-$ 100) did not activate transcription of the $\mathrm{UAS}_{\mathrm{G}_{\mathrm{G}}}-\mathrm{SNR6}$ reporter gene, and cells could not grow at $37^{\circ} \mathrm{C}$. (Plate d) The specific interaction between $\tau 138-G a l 11 \mathrm{Pp}$ and Gal4p(1-100) was able to activate expression of wild-type $\mathrm{U} 6$ snRNA from the $\mathrm{UAS}_{\mathrm{G}}$-SNR6 reporter gene and allow cell growth at $37^{\circ} \mathrm{C}$. 
Table 1. Leak Test for MPy11 Expressing Different $\tau 138 p$ Hybrid Proteins

\begin{tabular}{|lcc|}
\hline & \multicolumn{2}{c|}{$\begin{array}{c}\text { False Positives } \\
\text { in 108 Transformed Cells }\end{array}$} \\
\cline { 2 - 3 } Hybrid Protein & exp. 1 & exp. 2 \\
\hline$\tau 138-m B R C A 1$ (aa 1455-1812) & 0 & 3 \\
$\tau 138-m B A R D 1$ & 1 & 0 \\
$\tau 138-h S R B 7$ & 0 & 0 \\
$\tau 138-y G a l 11$ Pp & 0 & 1 \\
$\tau 138-y G a l 80 p$ & 0 & 0
\end{tabular}

The selectivity of our assay was tested by transforming the strain MPy 11 containing the $\mathrm{UAS}_{\mathrm{G}}-\mathrm{SNR} 6$ reporter plasmid with vectors expressing five different $\tau 138 \mathrm{p}$ hybrid proteins. For each $\tau 138 p$ hybrid protein, two independent experiments (exp. 1 and exp. 2) were performed. The observed growing colonies, the total number of which is presented for both sets of experiments, are referred to as false positives since no interaction partners fused to $G a l 4 p(D B D)$ were provided.

phenotype, thus allowing colony formation at $37^{\circ} \mathrm{C}$. A similar result was obtained by co-expressing two known interacting proteins (i.e., $\tau 138-\mathrm{Gal} 80 \mathrm{p}$ and Gal4p), which had been previously shown to specifically activate $\mathrm{UAS}_{\mathrm{G}^{-}}$ SNR6 transcription (10) (data not shown). To investigate sensitivity and specificity of our selection assay, we exploited the defined interaction between a fragment of the dimerization domain of Gal4p (residues 58-97) and Gal11Pp, a mutant form of the pol II holoenzyme component Gal11p carrying a single amino acid substitution (Asn342 to Ile) (1). From plasmon resonance experiments, this interaction is known to have a Kd of $10^{-7} \mathrm{M}$ (3). Since Gal4p only interacts with Gal11Pp but not with the wild-type form of Gal11p (Gal11p wt), these proteins provide an ideal model for testing the pol III two-hybrid selection system. Two $\tau 138$ fusion proteins were made, the first bearing Gal11p wt and the second bearing Gal11Pp. Equal levels of expression of these fusion proteins were detected by western blot analysis (data not shown). As a $\mathrm{UAS}_{\mathrm{G}}$-binding interaction partner, we expressed Gal4 $p$ (1-100), which contains the dimerization domain of Gal4p that interacts with Gal11Pp but not with Gal11p wt (2). Figure 2 shows that yeast ts cells expressing the fusion protein that contained the Gal11Pp mutation ( $\tau 138$ Gal11Pp) were able to grow at $37^{\circ} \mathrm{C}$, while no growth was detected for cells expressing the wild-type form of Gal11p fused to $\tau 138 \mathrm{p}$ ( $\tau 138-$ Gal11p wt) (plates $\mathrm{c}$ and d). The lack of growth at $37^{\circ} \mathrm{C}$ of yeast cells expressing $\tau 138-G a l 11 p$ wt is clearly dependent on the nonpermissive temperature because aliquots ( $1 / 3$ of the volume) of each transformation mixture were able to form colonies at $25^{\circ} \mathrm{C}$ (Figure 2, plate a).

Conditions mimicking a screening experiment were tested as follows: the plasmid expressing Gal4p(1-100) was mixed with its parental "empty" vector in defined ratios, and the derived plasmid mixture was used to transform the ts yeast strain that already contained the $\tau 138-G a 111 \mathrm{Pp}$ fusion protein described above. Only yeast cells expressing Gal4p (1-100) are expected to grow at $37^{\circ} \mathrm{C}$ due to the interaction with $\tau 138$ Gal11Pp that activates $\mathrm{UAS}_{\mathrm{G}}-\mathrm{SNR}$. According to the transformation efficiency for each individual plasmid in cells growing at $25^{\circ} \mathrm{C}$, the expected number of colonies expressing Gal4p (1100) was computed and referred to as $100 \%$. On the average, the actual num ber of colonies growing at $37^{\circ} \mathrm{C}$ was about $75 \%$ of this value. This difference is mostly due to a lower transformation efficiency of cells growing at the nonpermissive temperature of $37^{\circ} \mathrm{C}$ because the same type of experiment with a plasmid carrying the complete wildtype SNR6 gene gave similar values. Of all the colonies growing at $37^{\circ} \mathrm{C}, 60$ were randomly picked and subjected to 


\section{Short Technical Reports}

PCR analysis to assess the frequency of the Gal4p(1-100) plasmid. All of these cells contained the Gal4p(1-100) plasmid (data not shown). The recovery rate and the frequency of the Gal4p (1-100) plasmid indicate that our selection assay is very sensitive and tight.

To further investigate the selectivity of our assay, five $\tau 138 \mathrm{p}$ hybrid proteins (baits) were constructed, four of which carried protein domains that can activate transcription by pol II (data not shown), and tested for potential spontaneous suppression of the ts phenotype ("leak test"). To this purpose, these hybrid proteins were expressed in the ts strain containing the $\mathrm{UAS}_{\mathrm{G}}-\mathrm{SNR} 6$ reporter plasmid. For each $\tau 138$ p hybrid construct, and in two independent experiments, about $10^{8}$ transformed cells were plated and then incubated at $37^{\circ} \mathrm{C}$. Four days later, the number of growing colonies was counted (referred to as false positives). Zeros to a maximum of three false positives per $10^{8}$ transformed cells were observed (Table 1). We then compared the selectivity of our assay with the original 5-FOA method (10) by replica-plating cells expressing one of the $\tau 138$ p hybrid proteins ( $\tau 138$ Gal11Pp) on media containing $0.1 \% 5$ FOA and counting the number of grow ing colonies; application of the 5-FOA selection step gave an average of three false positives per $10^{4}$ transformed cells (i.e., $30000 / 10^{8}$ cells) (data not shown). Therefore, the selectivity of the pol III-based two-hybrid system was dramatically improved.

We have so far performed one screening experiment to completion with a mouse embryonic cDNA library fused to the DNA-binding Gal4p(1$147)$ and $\tau 138$-mBRCA 1 as a bait. For the pol III-based two-hybrid system, it is generally most convenient to fuse the bait protein to $\tau 138$ and the library to the Gal4p DNA-binding domain. Of a total of 2.5 million transformed cells, 272 colonies grew at $37^{\circ} \mathrm{C}, 188$ of which were confirmed by growth on $0.1 \%$ 5-FOA plates. After plasmid recovery, 183 of these 188 clones were shown to activate $\mathrm{UAS}_{\mathrm{G}}$-SNR6 in a bait-dependent manner. These 183 clones could be arranged in 14 complementation groups (details of these results will be published elsewhere). The results obtained through this series of screening experiments confirmed that our novel selection assay is indeed very efficient and reliable.

\section{REFERENCES}

1.Barberis, A. and L. Gaudreau. 1998. Recruitment of the RNA polymerase II holoenzyme and its implications in gene regulation. Biol. Chem. 379:1397-1405.

2.Barberis, A., J. Pearlberg, N. Simkovich, S. Farrell, P. Reinagel, C. Bamdad, G. Sigal, and M. Ptashne. 1995. Contact with a com ponent of the polymerase II holoenzyme suffices for gene activation. Cell 81:359-368.

3.Farrell, S., N. Simkovich, Y. Wu, A. Barberis, and M. Ptashne. 1996. Gene activation by recruitment of the RNA polymerase II holoenzyme. Genes Dev. 10:2359-2367.

4.Fields, S. and O. Song. 1989. A novel genetic system to detect protein-protein interactions. Nature 340:245-246.

5.Gietz, R.D., R.H. Schiestl, A.R. Willems, and R.A. Woods. 1995. Studies on the transformation of intact yeast cells by the LiAc/SSDNA/PEG procedure. Yeast 11:355-360.

6.Gietz, R.D. and A. Sugino. 1988. New yeastEscherichia coli shuttle vectors constructed with in vitro mutagenized yeast genes lacking six-base pair restriction sites. Gene 74:527534.
7.Kaiser, C., S. Michaelis, and A. Mitchell. 1994. Methods in Yeast Genetics. CSH Laboratory Press, Cold Spring Harbor, NY.

8.Madhani, H.D. and C. Guthrie. 1992. A novel base-pairing interaction between U2 and U6 snRNAs suggests a mechanism for the catalytic activation of the spliceosome. Cell 71:803-817.

9.Marsolier, M.C., N. Chaussivert, O. Lefebvre, C. Conesa, M. Werner, and A. Sentenac. 1994. Directing transcription of an RNA polymerase III gene via GAL4 sites. Proc. Natl. Acad. Sci. USA 91:11938-11942.

10.Marsolier, M.C., M.N. Prioleau, and A. Sentenac. 1997. A RNA polymerase III-based two-hybrid system to study RNA polymerase II transcriptional regulators. J. Mol. Biol. 268:243-249.

11.Marsolier, M.C., S. Tanaka, M. Livingstone-Zatchej, M. Grunstein, F. Thoma, and A. Sentenac. 1995. Reciprocal interference between nucleosomal organization and transcriptional activity of the yeast SNR6 gene. Genes Dev. 9:410-422.

12.Uetz, P., L. Giot, G. Cagney, T.A. Mansfield, R.S. Judson, J.R. Knight, D. Lockshon, V. Narayan et al. 2000. A comprehensive analysis of protein-protein interactions in Saccharomyces cerevisiae. Nature 403:623627.

13.Vidal, M. and P. Legrain. 1999. Yeast forward and reverse "n"-hybrid systems. Nucleic Acid Res. 27:919-929.

14.Walhout, A.J.M., R. Sordella, X. Lu, J.L. Hartley, G.F. Temple, M.A. Brasch, N. Thierry-Mieg, and M. Vidal. 2000. Protein interaction mapping in C. elegans using proteins involved in vulval development. Science 287:116-122.

We thank M.-C. Marsolier and A. Sentenac for discussion and for kindly providing yeast strains and plasmids, I. Irminger for the mouse BRCAl clone, and R. Bernards for the cDNA library. We also wish to thank L. Badi and D. Escher for comments on the manuscript and Walter Schaffner for support. Address correspondence to Dr. Alcide Barberis, ESBATech Inc., Winterthurerstr. 190, 8057 Zürich, Switzerland. e-mail: barberis@esbatech.com

Received 7 July 2000; accepted 11 September 2000.

\section{Michael Petrascheck ${ }^{1}$, Francesca Castagna ${ }^{1}$, and Alcide Barberis ${ }^{1,2}$ \\ ${ }^{1}$ University of Zürich \\ ${ }^{2}$ ESBATech, Inc. Zürich, Switzerland}

